\title{
Metabolic regulation in innate immunity
}

\begin{abstract}
The metabolic parameter dependent on the central nervous system (CNS) can regulate the storage and release of energy. Macrophages are the main effector cells that represent the innate immune system and have multiple roles, such as phagocytosis, secretion of cytokines, and antigen presentation. These responses are intervened by the generation of reactive oxygen/reactive nitrogen species (ROS/RNS), such as superoxide. In macrophages, G6PD (glucose 6-phosphate dehydrogenase) stimulates the expression of ROS- and RNS-producing genes. The immune response function requires rapid and extensive cell growth, proliferation, and production of effector cytokines. The metabolic and biosynthetic demand of lymphocytes increases after activation in relation to glucose metabolism via increasing glucose transporter 1. During the immune system response to pathogens, a special group of cytokines (chemokines) signals immune cells such as T-cells and macrophages to travel to the site of infection. These cytokines activate their cells to stimulate production of more cytokines. Many metabolic processes react directly or indirectly to proinflammatory cytokines to ensure a steady supply of nutrients for proliferation of phagocytes. This review has focused on energy metabolism regulation with neuroimmunity dependent cells.
\end{abstract}

Keywords: glucose, Immune system, toll like receptor
Volume 3 Issue 4 - 2018

\author{
Samin Ayromlou,' Leila Sadat Hatamnezhad, ${ }^{2}$ \\ Hormoz Ayromlou,' Siamak Sandoghchian \\ Shotorbani ${ }^{3}$ \\ 'Neurosciences Research Center, Tabriz University of Medical \\ Sciences, Iran \\ ${ }^{2}$ Department of Dermatology, Tabriz University of Medical \\ Sciences, Iran \\ ${ }^{3}$ Department of Immunology, Tabriz Branch, Islamic Azad \\ University, Iran
}

\section{Correspondence: Siamak Sandoghchian Shotorbani, Department of Immunology, Tabriz Branch, Islamic Azad University, Tabriz, Iran, Email siamak I331@gmail.com}

Received: June 12, 2018 | Published: July 23, 2018
Abbreviations: CNS, central nervous system; G6P, glucose 6-phosphate; GluT, glucose transferase; ATP, Adenosine three phosphate; NADPH, nicotinamide adenine dinucleotide phosphate; MAPKS, mitogen activated protein kinases; ROS/RNS, reactive oxygen/reactive nitrogen species; G6PD, glucose-6-phosphate

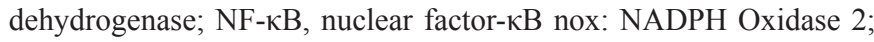
MIF, migration inhibitory factor; PKC, protein kinase C; TLR, tolllike receptors; PRRS, pattern recognition receptor; Myd88, myeloid differentiation primary response gene 88 ; TIR, toll/Interleukin-1 receptor; IRAK4, IL-1 receptor-associated kinase 4; TRAF6, TNF receptor associated factor; AP-1, activator protein 1 IKK: IאB kinase; JNK, c-Jun N-terminal kinases; TCR, T-cell receptor; TNF- $\alpha$, tumor necrosis factor- $\alpha$; Il-6, Interleukin-6; LIF, leukemia inhibitory factor; Il-1ß, Interleukin-1ß Il-1; Acp, The IL-1 accessory protein; Gp130, Glycoprotein 130; JAK, Janus kinase; STAT, signal transducer and activator of transcription; CRP, C-reactive protein.

\section{Introduction}

Metabolism is the chemical reaction that converts food into energy in the body. According to the first law of thermodynamics, energy cannot be created or destroyed; it has to be used or stored within biological systems. ${ }^{1}$ The metabolic processes in biological systems do not happen at random, but are tightly regulated to enable the most efficient use of the energy in ingested food. The metabolic parameter depends on the central nervous system (CNS), which can regulate the storage and release of energy. ${ }^{2}$ Metabolic regulation is modulated with enzyme activity. Glucose, one of the most important factors in metabolism, regulates the energy metabolism. ${ }^{3}$ Glucose 6-phosphate (G6P) is one of the glucose derivatives which is phosphorylated on carbon 6 and has two major metabolic pathways, glycolysis and pentose phosphate. ${ }^{4,5}$ Glucose 6-phosphate can also be converted to glycogen. This reaction is mediated through the enzymatic activity of hexokinase through the action of one molecule adenosine three phosphates (ATP). Glycogen can undergo a glycol genolysis reaction and form glucose-1-phosphate. Excessive production of Nicotinamide Adenine Dinucleotide Phosphate (NADPH) may lead to formation of
G6P by glucose 6-phosphate dehydrogenase(G6PD), which is the first step of the pentose phosphate pathway. ${ }^{4}$ In addition, if the body needs nucleotide precursors of DNA for growth and synthesis, G6P will also be dehydrogenated and enter the pentose phosphate pathway. ${ }^{6}$ The production of NADPH by G6PD is an essential enzyme in red blood cells, which carry oxygen from the lungs to tissues throughout the body. This enzyme helps protect red blood cells from damage and premature destruction. ${ }^{7}$ Many scientists have demonstrated the relationship between glucose and immune receptors and responses. Considine et al. ${ }^{9}$ verified the relationship between of high glucose inhibitions which can induce an inflammatory response. ${ }^{8}$ Also it has been confirmed that the macrophage infiltration depends on glucose levels in the body; they also demonstrated that some effectors could inhibit the glucose levels or G6PD genes and contribute to inflammatory diseases. ${ }^{9}$

\section{Metabolic regulation of cytokines}

The human immune system is energy intense and protection of immune functions has been thought to account for as much as $25 \%$ of the daily energy expenditure in healthy persons..$^{10}$ During the process of the immune system fighting pathogens, a special group of cytokines (chemokines) signals immune cells, such as T-cells and macrophages, to migrate to the site of infection. These cytokines can activate their cells and stimulate production of more cytokines. Many metabolic processes react directly or indirectly to proinflammatory cytokines to ensure a steady supply of nutrients for the proliferation of phagocyte cells and antibody production. The current hypothesis is that during an immune response, cytokines direct nutrients away from tissue growth. Therefore infection causes major modification in human metabolism. ${ }^{11}$ During sickness energy is reduced, fatty acid oxidation is increased to provide energy, and protein degradation is enhanced to supply amino acids for production of acute phase proteins. ${ }^{12}$ Some studies explained that the immune cells use glucose as their main fuel and also express the insulin receptor and respond to insulin. ${ }^{13}$ In addition, glutamine is essential for immune cell function and is highly used both as a primary fuel and as a carbon and nitrogen donor for nucleotide 
precursor synthesis. Also fatty acids using as a fuel but their oxidation does not useful for immune cell function. Proinflammatory cytokines such as IL-6, affect the metabolism of nutrients and also act in the brain to induce fever and physiological and behavioral changes which influence full energy balan. ${ }^{14}$ Adipokines such as tumor necrosis factor- $\alpha$ (TNF- $\alpha$ ), interleukin-6 (IL-6), leukemia inhibitory factor (LIF), and interleukin-1ß (IL-1ß) are involved in a wide range of physiological and metabolic processes synthesized and released from the adiposities and/or adipose tissue which have essential roles in body metabolism. ${ }^{15}$ The increase in inflammatory cytokine expression eventually increases the circulating levels of these proteins. ${ }^{16}$ Upon TNF release of from activated immunologically competent cells, enhanced glucose levels by these cells results (Figure 1). ${ }^{17}$

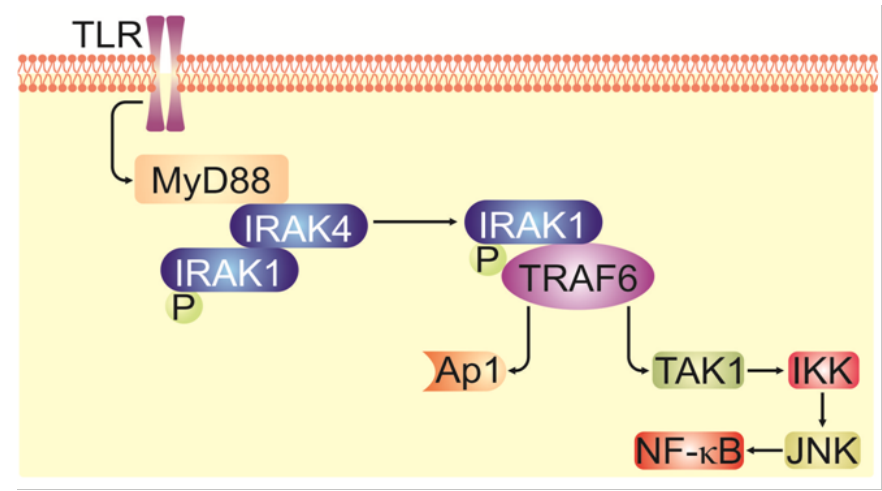

Figure I NF- $\kappa$ B pathway activation.

Glucose transfer into immune cells is mediated through GluT1 activity. ${ }^{18}$ TNF has been shown to enhance GluT1 expression and to increase GluT1 mediated glucose uptake. TNF decreases the uptake of glucose into the muscle and adipose cells governed by insulindependent Glut4 function. ${ }^{19}$ Active immune cells via secretion of TNF can be fueled with more glucose by inhibiting the uptake of glucose into muscle cells. This process can be regarded as a local energy request process. ${ }^{20}$ Proinflammatory cytokines such as IL-6 and members of the IL-1 family were initially identified as regulators of immune response. In normal immune cells a large amount of circulating IL-6 is produced in adipose tissue by macrophages. ${ }^{21}$ Stress and infections may cause high circulating levels in the body. However, cytokines play an important role in metabolic regulation. Increasing polymorphism in IL-6 and IL-1 variations are associated with both changes in activity and expression of these genes. IL-6 is an influential inducer of the acute phase response, and the acute phase reactant C-reactive protein is an important predictor and strong risk factor for metabolisms. Other proinflammatory cytokines, like IL-1ß and TNF $\alpha$, are also able to regulate the acute phase response. ${ }^{22}$

\section{Receptors and signal transduction}

The effect of cytokine on cells depends on cytokine levels, the profusion of the complementary receptor on the cell surface, and downstream signals activated by receptor binding. ${ }^{23}$ Each cytokine binds to an exact cell-surface receptor. Cell functions can be modified by following cascades of intracellular signaling. Redundancy is one of the characteristics of cytokines which causes cytokines appear to share receptor subunits and exert similar functions. ${ }^{24}$ For glucose transportation in the immune system, the IL-1RI complex, which is composed of the IL-1RI and the IL-1 accessory protein (IL-1AcP), is necessary. This complex is responsible for signal transduction. ${ }^{25}$
IL-1RI with its accessory protein is followed by recruitment and phosphorylation of the IRAK via the docking molecule MyD88, leading to NF- $\kappa$ B activation. ${ }^{26} \mathrm{IL}-1 \mathrm{a} / \mathrm{B}$ and IL-1 receptor antagonist act through a heterodimer consisting of the IL-1RI and the IL-1 accessory protein (IL-1AcP). Binding of agonists induces the recruitment of MyD88 and initiates the activation of IRAK/TRAF pathway, leading to NF- $\kappa \mathrm{B}$ activation. ${ }^{27}$ The IL-6 cytokine family, including IL-6, IL11, and LIF, shares the glycoprotein 130 (gp130) signal transducer and signal through gp130 and a ligand specific receptor. Although the gp130 expresses across all cell types, it cannot transduce signals without the ligand specific receptor. The IL-6 cytokine family shares the gp130 signal transducer and signal through gp130 and a ligand specific receptor. ${ }^{28}$ The IL-6/IL-6Ra fusion-protein Hyper-IL-6 can activate all cells with gp130 even in the absence of the ligand specific receptor. Binding of agonists induces phosphorylation of JAK and initiates the activation of JAK/STAT or JAK/MAPK pathway.

\section{Conclusion}

Metabolism is the chemical reaction that converts food to energy which can be regulated by the central nervous system. Brain function is linked to glucose infiltration and transportation of energy, which can be altered by cytokines and proinflammatory cytokines via the immune system. Glucose infiltration in the immune system can manage innate immunity by regulating the complement cascades and also by producing adaptive immunity components. Energy metabolism has a major relation with complement as an important part of innate immunity. As a final remark, further investigations are needed to provide more evidence to show the relationship between energy metabolism and occurrence of CNS immune diseases.

\section{Acknowledgments}

We are thanks to Dr. Javad Mahmoudi, Dr. Leila Sadat Hatamnezhad and Dr. Sandoghchian supporting in this rewive.

\section{Conflict of interest}

Author declares there is no conflict of interest.

\section{References}

1. Auernhammer C, Melmed S. Leukemia-inhibitory Factor-neuroimmune Modulator of Endocrine Function. Endocr Rev. 2000;21(3):313-345.

2. Besedovsky HO, Del Rey A. Immune-neuro-endocrine interactions: facts and hypotheses. Endocr Rev. 1996;17(1):64-102.

3. Beutler B, Mahoney J, Le Trang N, et al. Purification of cachectin, a lipoprotein lipase-suppressing hormone secreted by endotoxin-induced RAW 264.7 cells. J Exp Med. 1985;161(5):984-995.

4. Bittencourt J, Presse F, Arias C, et al. The melanin-concentrating hormone system of the rat brain: An immuno-and hybridization histochemical characterization. J Comp Neurol. 1992;319(2):218-245.

5. Cao Z, RM Umek, Mc Knight SL. Regulated expression of three C/ EBP isoforms during adipose conversion of 3T3-L1 cells. Genes Dev. 1991;5(9):1538-1552.

6. Chida D, Osaka T, Hashimoto O, et al. Combined interleukin-6 and interleukin-1 deficiency causes obesity in young mice. Diabetes. 2006;55(4):971-977.

7. Chua SC, Chung WK, Wu Peng XS, et al. Phenotypes of mouse diabetes and rat fatty due to mutations in the OB (leptin) receptor. Science. 1996;271(5251):994-996. 
8. Chung J, Uchida E, Grammer TC, et al. STAT3 serine phosphorylation by ERK-dependent and-independent pathways negatively modulates its tyrosine phosphorylation. Mol Cell Biol. 1997;17(11):6508-6516.

9. Considine RV, Sinha MK, Heiman ML, et al. Serum immunoreactiveleptin concentrations in normal-weight and obese humans. $N$ Engl J Med. 1996;334(5):292-295.

10. El Haschimi K, Pierroz DD, Hileman SM, et al. Two defects contribute to hypothalamic leptin resistance in mice with diet-induced obesity. J Clin Invest. 2000;105(12):1827-1832.

11. Ericsson A, Liu C, Hart R, et al. Type 1 interleukin-1 receptor in the rat brain: Distribution, regulation, and relationship to sites of IL-1-induced cellular activation. J Comp Neurol. 1995;361(4):681-698.

12. Fajas L, Landsberg RL, Huss Garcia Y, et al. E2Fs regulate adipocyte differentiation. Dev Cell. 2002;3(1):39-49.

13. Farooqi IS, Jebb SA, Langmack G, et al. Effects of recombinant leptin therapy in a child with congenital leptin deficiency. $N$ Engl J Med. 1999;341(12):879-884.

14. Fingerle Rowson G, Koch P, Bikoff R, et al. Regulation of macrophage migration inhibitory factor expression by glucocorticoids in vivo. $\mathrm{Am} \mathrm{J}$ Pathol. 2003;162(1):47-56.

15. Fischer MJ, Goldschmitt C, Peschel JP, et al. A bioactive designer cytokine for human hematopoietic progenitor cell expansion. Nat Biotechnol. 1997;15(2):142-145.

16. Fu M, Rao M, Bouras T, et al. Cyclin D1 inhibits peroxisome proliferatoractivated receptor gamma-mediated adipogenesis through histone deacetylase recruitment. J Biol Chem. 2005;280(17):16934-16941.

17. García MC, Wernstedt I, Berndtsson A, et al. Mature-onset obesity in interleukin-1 receptor I knockout mice. Diabetes. 2006;55(5): 1205-1213.

18. Gayle D, Ilyin SE, Plata Salamán CR. Feeding status and bacterial LPSinduced cytokine and neuropeptide gene expression in hypothalamus. $\mathrm{Am}$ J Physiol. 1999;277(4):1188-1195.
19. Gayle D, Ilyin SE, Romanovitch AE, et al. Basal and IL-1beta-stimulated cytokine and neuropeptide mRNA expression in brain regions of young and old Long-Evans rats. Brain Res Mol Brain Res. 1999;70(1):92-100.

20.Gregersen PK, Bucala R. Macrophage migration inhibitory factor, MIF alleles, and the genetics of inflammatory disorders: incorporating disease outcome into the definition of phenotype. Arthritis Rheum. 2003;48(5):1171-1176

21. Halaas JL, Gajiwala KS, Maffei M, et al. Weight-reducing effects of the plasma protein encoded by the obese gene. Science. 1995;269(5223):543546.

22. Hirano T. Interleukin 6 and its receptor: ten years later. Int Rev Immunol. 1998;16(3-4):249-284.

23. Hisashi Y, Masabumi M, Masamichi S. Localization of mRNA for leukemia inhibitory factor receptor in the adult rat brain. J Neuroimmunol. 1996;70(1):45-53.

24. Hotamisligil GS, Arner P, Caro JF, et al. Increased adipose tissue expression of tumor necrosis factor-alpha in human obesity and insulin resistance. $J$ Clin Invest. 1995;95(5):2409-2415.

25. Hotamisligil GS, Murray DL, Choy LN, et al. Tumor necrosis factor alpha inhibits signaling from the insulin receptor. Proc Natl Acad Sci U S A. 1995;91(11):4854-4858.

26. Hotamisligil GS, Peraldi P, Budavari A, et al. IRS-1-mediated inhibition of insulin receptor tyrosine kinase activity in TNF- $\alpha$-and obesity-induced insulin resistance. Science. 1996;271(5249):665-670.

27. Hotamisligil GS, Shargill NS, Spiegelman BM. Adipose expression of tumor necrosis factor-alpha: direct role in obesity-linked insulin resistance. Science. 1993;259(5091):87-91.

28. Iwata M, Haruta T, Usui I, et al. Pioglitazone ameliorates tumor necrosis factor- $\alpha$-induced insulin resistance by a mechanism independent of adipogenic activity of peroxisome proliferator-activated receptor- $\gamma$. Diabetes. 2001;50(5):1083-1092. 